\title{
La educación estadística a la luz de la educación matemática crítica
}

\section{Statistical Education in the Light of Critical Mathematics Education}

A educação estatística à luz da educação matemática crítica

Felipe Jorge Fernández-Hernández* iDorcid.org/0000-0003-3775-4757

Luisa Andrade-Escobar** (iD orcid.org/0000-0002-1168-1468

Para citar este artículo

Fernández-Hernández, F. y Andrade- Escobar, L. (2021). La educación estadística a la luz de la educación matemática crítica. Revista Colombiana de Educación, 1(83), 1-20. https://doi.org/10.17227/rce.num83-10772.

Fecha de recepción: 26/11/2019

Fecha de evaluación: 06/10/2020

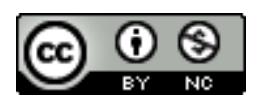

* Matemático. Profesor de planta Universidad Pedagógica Nacional, Bogotá, Colombia. Correo: fjfernandez@pedagogica.edu.co

** Matemática. Profesora Universidad Pedagógica Nacional, Bogotá, Colombia. Correo: luandradeescobar@gmail.com 


\section{Resumen}

\author{
Palabras clave: educación \\ estadística; alfabetización; \\ cultura; razonamiento y \\ pensamiento estadístico; \\ educación matemática crítica
}

En una revisión de la investigación en educación estadística desde disertaciones recientes, se encuentra extensa indagación en torno a la alfabetización, cultura, razonamiento y pensamiento estadístico, así como evidencias de la tendencia de la comunidad internacional a incorporar la teoría crítica en la educación. En este artículo, se presenta una exploración acerca de posibles relaciones y convergencias entre principios de la educación matemática crítica y lineamientos de la educación estadística, que propicia la búsqueda de propuestas en conjunto y ofrece ideas promisorias tanto para la enseñanza-aprendizaje como para la investigación en estadística. Además, pretende la búsqueda de propuestas en conjunto para integrar las dimensiones sociales, culturales y políticas en las prácticas educativas de la estadística, y así contribuir a la formación de ciudadanos participativos de la sociedad.

\section{Abstract}

\author{
Keywords: statistical \\ education; statistical literacy; \\ culture; statistical reasoning \\ and thinking; critical \\ mathematics education
}

In a review of statistical education research from recent dissertations, one finds extensive inquiry into literacy, culture, reasoning, and statistical thinking, as well as evidence of the international community's tendency to incorporate critical theory into education. In this article, an exploration of possible relationships and convergences between principles of technical mathematics education and guidelines of statistical education is presented, which encourages the search for joint proposals and offers promising ideas both for teaching-learning and for education research in statistics. In addition, it seeks to search for joint proposals to integrate the social, cultural, and political dimensions in the educational practices of statistics to contribute to the formation of participatory citizens of society.

\section{Resumo}

Em uma revisão da pesquisa em educação estatística, a partir de dissertações recentes, encontra-se uma extensa

Palavras-chave: educação

estatística; alfabetização;

cultura; raciocínio e

pensamento estatístico;

educação matemática crítica indagação sobre a alfabetização, cultura, razonamento e pensamento estatístico, bem como evidências da tendência da comunidade internacional de incorporar a teoria crítica na educação. Neste artigo, apresenta-se então uma exploração sobre possíveis relações e convergências entre princípios da educação matemática crítica e diretrizes da educação estatística, que propicia a busca das propostas em conjunto e oferece ideias promissoras tanto para o ensinoaprendizagem, quanto para a investigação estatística. Além disso, considera a busca de propostas em conjunto para integrar as dimensões sociais, culturais e políticas nas práticas educacionais da estatística, e assim, contribuir para a formação de cidadãos participativos da sociedade. 


\section{Introducción}

Resultados relevantes de proyectos anteriores (Andrade et al., 2017; Fernández et al., 2016; Fernández et al., 2018) muestran la vigencia del trabajo con la alfabetización, cultura, razonamiento y pensamiento estadístico en estudios recientes de educación estadística. Por ejemplo, las investigaciones de Weber (2017), Le (2017), Sabbag (2016), Ziegler (2014), así como la inserción de elementos de la perspectiva crítica en diversas disertaciones [ver, por ejemplo, Kelly (2019), Campos (2007), Peters (2009), Hassad (2007)].

Con el fin de examinar una posible cercanía entre la educación estadística y la educación matemática crítica (EMC) como campos del saber, se decide, entonces, indagar más a fondo en el paradigma crítico en la educación, en particular en el enfoque de la EMC y en el estado de elaboración de la alfabetización, cultura, razonamiento y pensamiento estadístico, como metas de enseñanza y aprendizaje. Para esto, se recopila literatura en un proceso sistemático de búsqueda y organización de documentación según las recomendaciones de Dulzaides y Molina (2004); luego, se consulta la bibliografía acopiada y se identifican aspectos constitutivos de cada campo para así caracterizarlo; posteriormente, se contrastan tales aspectos $\mathrm{y}$ en estos cruces se determinan posibles convergencias que permiten aislar elementos comunes para constituir categorías que contribuyen a la comprensión, en el sentido señalado por Denzin y Lincoln (2015).

En este artículo, para comenzar, se introduce la educación estadística y se incluyen breves descripciones de la alfabetización, cultura, razonamiento, pensamiento y sentido estadístico, con los principales lineamientos que los caracterizan, para proveer una mirada inicial de cada uno de estos constructos ${ }^{1}$. Luego, se describen fundamentos de la EMC, sin pretensiones exhaustivas que, también, suministran una primera mirada de esta disciplina. Estas miradas se amplían y complementan con los aspectos y nociones adicionales que se discuten en la siguiente sección, donde se exponen y contrastan ideas de las dos disciplinas que parecen ir en la misma dirección.

Al finalizar, se sintetizan las resonancias encontradas, que se perciben como promisorias para enriquecer la educación estadística y avanzar en la formación democrática de ciudadanos y que, además, pueden ser constitutivas de una visión crítica de la educación estadística.

\section{La educación estadística}

Señala Moore $(1992 ; 1998 ; 1999)$ que la estadística es una epistemología en sí misma y no una rama de las matemáticas. La naturaleza incierta, inductiva y subjetiva propia de la estadística (Eichler y Zapata, 2016) involucra formas poderosas y particulares de pensar, la separa de las matemáticas y genera el desafío de establecer y definir sus características únicas. Con el mismo ángulo, Del Pino y Estrella (2012) describen algunas diferencias conceptuales que involucran competencias y desarrollos de pensamiento particulares a la estadística, tales como la orientación conceptual enfocada en la variabilidad que le da a la

\footnotetext{
1 Un constructo es una construcción teórica, un objeto conceptual que implica una correspondencia con procesos mentales.
} 
estadística un carácter distintivo, la interdisciplinadidad inherente a esta disciplina y la ineludible visibilidad de los contextos de los datos.

La educación estadística enfrenta sus propios dilemas, distintos de los de la educación matemática. Por ejemplo, comprender el aprendizaje de la inferencia estadística al utilizar herramientas particulares de la estadística como la simulación, conjuntos de datos reales y visualizaciones dinámicas (Batanero, 2019).

Los investigadores en educación estadística se preocupan por describir, caracterizar y a su vez interrelacionar, las formas de pensar específicas de la estadística. En este sentido, existe un trabajo serio y amplio en torno a la alfabetización, cultura, razonamiento y pensamiento estadístico como formas de razonar que se pretenden alcanzar. Numerosos estudios procuran distinguir los procesos, habilidades y competencias involucrados en el desarrollo de cada una, para así promoverlos en los estudiantes y formar ciudadanos competentes en estadística (Garfield y Ben-Zvi, 2008; Budget y Pfannkuch, 2007; delMas, 2004; Gal, 2004; 2016; Chance, 2002; Watson, 2006).

Aunque la comunidad académica continúa debatiendo y precisando estos constructos (Rumsey, 2002; Jablonka, 2003; Ben-Zvi y Garfield, 2004; Garfield et al., 2010; Sabbag, 2016) en foros de investigación como el Statistical Reasoning, Thinking and Literacy Research Forums (SRTL, http://srtl.stat.auckland.ac.nz/), las caracterizaciones que se presentan a continuación son las más conocidas y trabajadas.

\section{Alfabetización estadística}

Aunque, como dice Rumsey (2002), la "alfabetización estadística" es una expresión muy amplia donde caben diversidad de definiciones, es claro que sugiere la idea de una cultura básica o primaria relativa a la estadística como base de la enseñanza estadística.

Así, la caracterización de la alfabetización estadística de Garfield y Ben-Zvi (2008) y BenZvi y Garfield (2004) incluye el desarrollo de competencias y habilidades básicas utilizadas en la comprensión de información estadística, como leer y organizar datos, elaborar tablas, representar datos en diferentes registros, comprender conceptos, vocabulario, símbolos y la probabilidad como medida de incertidumbre. De manera similar, en el Guidelines for Assessment and Instruction in Statistics Education (GAISE, 2016), la alfabetización estadística es definida como comprender conceptos claves, símbolos y lenguaje estadístico. delMas (2004) concreta palabras y verbos particulares que permiten evidenciar el desarrollo de alfabetización, razonamiento y pensamiento estadístico.

Todos estos autores, lo mismo que Chance (2002) y Rumsey (2002), asumen intersecciones entre la alfabetización, el razonamiento y el pensamiento estadístico, y en consecuencia en su desarrollo; aún más, la alfabetización estadística se considera el cimiento del razonamiento y el pensamiento. También, para Budget y Pfannkuch (2007), la alfabetización estadística abarca elementos del razonamiento y pensamiento estadístico.

\section{Cultura estadística}

La "cultura estadística" de Batanero (2001), descrita como "algo más que capacidad de cálculo y conocimiento de definiciones", se equipara a la alfabetización estadística que se ha expuesto, pues, de acuerdo con Batanero $(2001 ;$ 2002) y Batanero et al. (2013), involucra conocer conceptos y procedimientos de la estadística, comprender ideas de diseño de experimentos, 
recolección y representación de datos, incertidumbre, probabilidad y riesgo, comparar representaciones, proporcionar y justificar conclusiones y predicciones. Sin embargo, la mirada al desarrollo de cultura estadística se enmarca en la perspectiva semiótica que estos investigadores trabajan, y, por lo tanto, requiere de procesos interpretativos que ponen en relación diversos tipos de objetos (expresiones del lenguaje, conceptos, propiedades, acciones, argumentos) mediante correspondencias de tipo semiótico (Godino et al., 2007); además, en la cultura estadística tercian las intuiciones y componentes emocionales.

\section{Razonamiento estadístico}

Para Garfield y Ben-Zvi (2008), el razonamiento estadístico abarca la alfabetización estadística y está constituido por la forma en que se razona en torno a ideas estadísticas y se le da sentido a la información estadística; involucra interpretar datos con base en representaciones o resúmenes estadísticos, relacionar conceptos y conectar ideas acerca de datos y azar; es decir, una persona que razona estadísticamente entiende y explica procesos estadísticos, y comprende e interpreta resultados estadísticos. En palabras de delMas (2004), el razonamiento estadístico se demuestra cuando se explica y justifica por qué es apropiado un modelo o representación particular, y por qué ocurre un determinado resultado. Añaden Garfield y Ben-Zvi (2008), que el razonamiento estadístico se percibe en las representaciones y conexiones de los conceptos estadísticos.

\section{Sentido estadístico}

Batanero et al. (2013) denominan "sentido estadístico" a la cultura estadística y el razonamiento estadístico en conjunto, denominación que tiene una connotación similar al "sentido numérico" de la educación matemática, que considera tanto el conocimiento como los razonamientos implicados.

\section{Pensamiento estadístico}

El pensamiento estadístico involucra, según delMas (2004), Garfield y Ben-Zvi (2008), y Pfannkuch y Wild (2004), además de la alfabetización y el razonamiento estadístico, comprender, utilizar e integrar el contexto del problema en investigaciones, extraer conclusiones, reconocer y entender el proceso completo de investigación. Igualmente, incluye reflexionar sobre técnicas de análisis de datos, resúmenes numéricos y representaciones visuales, la naturaleza del muestreo para hacer inferencias, el diseño de experimentos que establece causalidad, la simulación de fenómenos aleatorios con modelos estadísticos y probabilísticos, el uso de herramientas de inferencia estadística para el proceso investigativo, tener en cuenta la variación y la transnumeración. En esencia, para Ben-Zvi y Garfield (2004), el pensamiento estadístico posibilita evaluar y criticar resultados de un problema o de un estudio estadístico.

\section{La educación matemática crítica}

La educación ha sido uno de los intereses de la teoría crítica que emergió en el siglo xx, donde no solo es vista como un mecanismo de reproducción de las estructuras sociales y económicas, sino también es concebida como un espacio de reacción y resistencia (Valero, 1999). Dice Skovsmose (2016) que el sentido de una educación crítica es abordar discursos con 
información errada, estereotipos y supuestos, cualquier forma de opresión, explotación, exclusión e injusticias sociales, con el fin de abrir posibilidades a cambios sociales.

En particular, sostiene Skovsmose (2014) que una educación matemática que sea crítica, puede ser caracterizada "en términos de la preocupación con respecto a los diferentes roles socio-políticos que la matemática en acción y la educación matemática podrían jugar". La aproximación, conocida como educación matemática crítica, se origina en la década de 1980, por el giro que se realiza en ese periodo "hacia los aspectos sociales en la educación matemática" (Valero et al., 2015). En ese entonces, las afirmaciones de que las matemáticas y su educación están relacionadas con la cultura, y aún más con la democracia, la política y el poder, causaron desconcierto e incluso rechazo. No obstante, aseveran Valero et al. (2015), que el tiempo transcurrido y las políticas educativas mundiales que han asociado el rendimiento en matemáticas con la efectiva participación de los ciudadanos en la vida social, económica y política han generado aceptación de esta aproximación por parte de maestros e investigadores.

Aguilar (2014) resalta que desde su inicio el foco de la EMC ha sido cómo la sociedad hace uso de las matemáticas y las consecuencias de tal uso. Para Skovsmose (1999b; 2012a; 2012b; 2014; 2016), la EMC es, entonces, una expresión de preocupaciones amplias sobre la educación matemática, como trabajar por la justicia social y en contra de la exclusión y supresión social, abrir nuevas posibilidades para los estudiantes y abordar críticamente los usos de las matemáticas en todas sus formas y aplicaciones.

Esto supone un cambio en las herramientas conceptuales para estudiar la educación matemática y una exensión del propósito mismo de la investigación, para ir más allá de generar propuestas de mejora de la enseñanza y el aprendizaje de las matemáticas a asuntos como la comprensión de los fenómenos de la educación matemática, de sus problemáticas y de sus relaciones con la sociedad y la democracia (Valero et al., 2015). Se marcan así, según Valero et al., al menos tres direcciones de trabajo: "La crítica a las matemáticas en la sociedad; la relación entre educación matemática y democracia, justicia social, equidad e in(ex)clusión; y la invención de nuevas posibilidades educativas" (2015, p. 290).

En la EMC, la discusión de la educación no es solo alrededor de preparar para la educación futura o el mercado laboral, sino también acerca de preparar para la ciudadanía y participación en la vida social y política. En consecuencia, uno de sus principios es el ejercicio de la democracia en las prácticas del aula de matemáticas para favorecer el desarrollo de una ciudadanía democrática y crítica (Skovsmose, 1999b; Skovsmose y Borba, 2004). Así, en el proceso de enseñanza y aprendizaje de las matemáticas, se destacan cuestiones como los aspectos sociales y políticos del aprendizaje de las matemáticas y el uso y la función de las matemáticas en la vida diaria o en aplicaciones avanzadas (Skovsmose y Borba, 2004), la importancia de las matemáticas para el desarrollo científico-tecnológico que hace parte del desarrollo social (Vithal y Skovsmose, 2012).

De acuerdo con estos asuntos, la investigación en EMC pone atención a cuestiones problemáticas que han sido señaladas como relevantes para la educación matemática. Algunas de ellas son: el contexto social de los niños, la clase multilingüe y multicultural, la organización del proyecto de trabajo en educación matemática, la confianza y fiabilidad de las matemáticas en la práctica, la convicción en la idea de seguridad de las matemáticas, la distribución de los recursos y el acceso a la tecnología (Skovsmose, 2012b). 


\section{Hacia una educación crítica en estadística}

A pesar de que la investigación en educación estadística, por razón de la atención que la estadística y probabilidad reciben en los currículos de la escuela y la universidad, ha ido evolucionando (Batanero, 2019), se requiere que su desarrollo esté dirigido a atender las necesidades específicas de cada colectividad. En esta dirección parece pertinente poner el acento en la formación ciudadana y, por lo tanto, ensanchar la mirada de la educación estadística desde ángulos del paradigma crítico, en particular de la EMC. Las ideas examinadas de las dos disciplinas se organizan a continuación en las categorías emergentes en el proceso.

\section{Universalidad}

Ya desde comienzos de este siglo, Clements (2000) señala que la investigación en torno a la equidad en la educación ha tenido el objetivo de trascender el sentido usual de equidad, para interpretarlo a partir de la propuesta de la Unesco: equidad en el acceso, en el trato, en el proceso y en los resultados. Con la misma orientación, Skovsmose (1999a) explicita como deber primordial de la democracia proveer la igualdad de oportunidades, es decir, proporcionar las condiciones para que cualquiera pueda perseguir sus intereses en cooperación y consenso con otros. Skovsmose y Valero (2012a) agregan que una democracia propende por una distribución justa de los servicios sociales y, por consiguiente, debe garantizar que cada individuo tenga igualdad de acceso a la escolaridad y al aprendizaje.

En particular, la EMC establece como uno de sus principios el ejercicio de la democracia con el compromiso ineludible del acceso y receptividad de las ideas matemáticas para todos, independientemente del color de piel, género y clase (Skovsmose, 1999b; Skovsmose y Borba, 2004). Esta postura incluye la educación matemática para la justicia social y una educación matemática que sea receptiva por todos (Skovsmose, 1999b; 2016). En forma similar, Cobb (2005) señala el proceso de democratización acaecido, y en marcha, de la educación estadística, donde se han ampliado las trayectorias, los intereses y las motivaciones de las personas que aprenden estadística, $y$, en consecuencia, las ideas estadísticas han pasado de ser enseñadas a un grupo pequeño de futuros científicos, a ser enseñadas a estudiantes de muchos niveles con intereses y propósitos diversos.

En la EMC, Skovsmose (1999a) plantea la necesidad de alfabetización matemática -en inglés mathemacy o matheracy - en un sentido cercano a la concepción de alfabetización de Freire (1967), que alude a una competencia requerida para todos, que posibilita leer el mundo y comprender su contexto. Valero et al. (2015) destacan la preocupación de la EMC por lograr que esta alfabetización matemática sea una de las competencias más importantes de todo ciudadano.

De manera parecida, alfabetización estadística alude al "hecho de que la estadística se considera ahora como parte de la herencia cultural necesaria para el ciudadano" (Batanero et al., 2013). Desde mitad del siglo pasado, Huff (1954) menciona las palabras del escritor Wells que auguran que un día "pensar estadísticamente será tan necesario para el ciudadano eficiente como leer y escribir". Al respecto, Batanero (2019) manifiesta que las indagaciones en educación estadística están en la actualidad bien establecidas para responder a la necesidad de alfabetizar en estadística a todos los ciudadanos. Así, Garfield y Ben-Zvi (2008) corroboran que la alfabetización estadística alude a un proceso y un logro que se persigue para todos como resultado de la escolaridad, por ser necesario, e incluso imperativo, para el diario vivir 
de toda persona en la sociedad actual, de igual forma que lo que ocurre con la alfabetización concerniente a la lectura y escritura del lenguaje.

Añade Huff (1954) que la función principal de la enseñanza de la estadística en la escuela es el proceso de alfabetización estadística, el cual debe formar a la población para pensar estadísticamente. Igualmente, Ben-Zvi y Garfield (2004) y Garfield y Ben-Zvi (2008), apuntan que la educación debe formar a todos los estudiantes para entender información cuantitativa y evaluar evidencias y afirmaciones basadas en datos, que afectan asuntos importantes de sus vidas.

\section{El aula como espacio de la vida real}

Señala Spinoza (1999) que la sociedad es una expresión de la actividad en la escuela, pues en las prácticas escolares emerge un carácter social, cultural y político que necesariamente envuelve toda situación trabajada en el aula; es allí donde se construye sociedad y se producen espacios de lo público.

Así, para la EMC las matemáticas son un lenguaje o herramienta poderosa que crea realidades y da forma a nuestras sociedades con su poder formativo (Valero et al., 2015). Anota Skovsmose (2014) que el proceso educacional propuesto por la EMC posibilita compartir experiencias en torno a problemáticas ligadas a la realidad social entre todos los actores, que "es primordial que los problemas tengan que ver con situaciones y conflictos sociales fundamentales", y que "es esencial que los estudiantes puedan al mismo tiempo reconocer esos problemas como sus propios problemas". El trabajo en torno a las matemáticas en la EMC favorece el debate alrededor de la función y utilidad de las matemáticas en la vida diaria o en aplicaciones avanzadas (Skovsmose y Borba, 2004).

Parte de la investigación crítica en la EMC está en generar un diálogo para darle vida a situaciones de enseñanza y aprendizaje inmersas en contextos reales relevantes para los estudiantes (Skovsmose y Borba, 2004).

Por su parte, el objetivo del trabajo en estadística es abordar situaciones de la realidad. Para Chance (2002), el trabajo con la estadística, por razón de su esencia, es siempre dependiente de unos datos, los cuales típicamente están arraigados en un contexto (Moore, 1998; Pfannkuch y Wild, 2004). Afirma Moore (1999) que la estadística es datos en contexto. En la misma dirección, Chance (2002) destaca la importancia en la estadística del contexto del cual surgieron los datos y para el que se aplicarán los hallazgos; Cobb y Moore (1997, citados en Pfannkuch y Wild, 2004) advierten que el conocimiento del contexto es esencial, porque contribuye a decidir si los patrones encontrados en los datos tienen significado y cómo se vinculan con la situación; en palabras de Scheaffer (1997), involucrarse en el enunciado del problema ayuda a interpretar los datos en el contexto.

También la educación estadística, que no ha sido ajena a las recomendaciones de reformas al currículo de matemáticas sobre el trabajo con situaciones cotidianas y con datos reales familiares a los estudiantes (Ministerio de Educación Nacional [MEN], 1996; National Council of Teachers of Mathematics (NCTM), 2000; GAISE, 2016), enfatiza el abordaje de problemas en contextos reales; pues, como dice delMas (2004), el desarrollo de pensamiento estadístico en la escuela ocurre dentro de situaciones concretas. La importancia del trabajo estadístico escolar en contextos de la sociedad también es resaltada por Gal (2004) y Watson (2006), al reconocer que la educación estadística ofrece una forma de abordar los problemas de la realidad que permite entender el entorno social; así, Magallanes et al. (2013) apuntan que el 
contexto de la situación no opera simplemente como decoración, sino que genera el aprendizaje y se explica a través de este.

En referencia a la enseñanza y el aprendizaje de la estadística, el mismo Skovsmose (2016) indica que muchas situaciones problemáticas de la vida real pueden abordarse a través de la investigación, donde el foco de los procedimientos estadísticos es el problema que se trata. En igual dirección, Batanero et al. (2013) y English (2013) sugieren que la enseñanza de la estadística basada en el trabajo con investigaciones en torno a situaciones de la vida real, "planteadas por el profesor o escogidas libremente por los alumnos" posibilita relacionar los diversos objetos estadísticos con asuntos de la realidad y por lo tanto una mejor comprensión de estos, es decir, favorece el desarrollo de alfabetización y sentido estadístico.

Análogamente, Azcarate y Cardeñoso (2011) vinculan la alfabetización estadística con el trabajo a partir de datos extraídos de las situaciones que los ciudadanos enfrentan cotidianamente, y Budgett y Pfannkuch (2007) consideran como parte de la alfabetización estadística el conocimiento de los eventos del día a día desde una perspectiva estadística.

\section{Postura frente a las matemáticas y la estadística}

La EMC, como muchos otros enfoques teóricos, ha incluido la discusión acerca de la necesidad de adoptar una postura frente a las matemáticas. Señala Valero (2012) que la neutralidad de las matemáticas y de la educación matemática está en tela de juicio, pues ambas forman parte de las tecnologías de poder de las sociedades y, en consecuencia, influyen en decisiones que tienen repercusiones en la vida de las personas. Aún más, Valero et al. (2015) argumentan que los modelos matemáticos que sustentan sistemas científicos y tecnológicos desarrollados para el progreso y bienestar de la humanidad, también han implicado generación de estructuras de riesgo y catástrofes.

La EMC propone trascender la reflexión ceñida a la clase de matemáticas, reconociendo e incorporando dimensiones del ámbito sociopolítico en el que se desarrollan las clases (Scaglia, 2012). Así, desde sus comienzos, el foco de la EMC ha sido "cómo la sociedad hace uso de las matemáticas, las consecuencias de tal uso y las implicaciones de las matemáticas como parte de las prácticas educativas" (Valero et al., 2015).

En la propuesta de la EMC, Skovsmose (2010) considera las matemáticas como una racionalidad que es crítica porque es significativa, dado que impacta todas las esferas de la vida social, y es indeterminada, puesto que puede encaminarse en cualquier dirección. A partir de precisar la expresión "matemática en acción" como los procesos donde las ideas matemáticas abstractas son proyectadas en la realidad ${ }^{2}$, Skovsmose (2010) fortalece sus argumentos sobre la indeterminación de las matemáticas como racionalidad, y señala que "como cualquier acción, la matemática en acción puede ser discutida en un rango de cualidades. Tales acciones pueden ser beneficiosas, costosas, sorprendentes, arriesgadas, aburridas, etc.". Sostiene, entonces, Skovsmose (2004) que los papeles que juegan las matemáticas en la formación de la sociedad no son fijos, están "disponibles para ser actuados a través de la educación matemática" y "es por esto que la educación matemática es crítica".

Esta característica crítica de la EMC, de acuerdo con Skovsmose y Valero (2012a, p. 00), la aleja de ideas y justificaciones que a lo largo del tiempo han abogado por el valor inherente de las matemáticas en la escolaridad, y, contribuye "a entender cómo el conocimiento

\footnotetext{
2 Estos procesos son inversos a la abstracción, en la que se extraen ideas matemáticas de la observación empírica.
} 
matemático y el conocimiento de las matemáticas escolares se imbrican en y con la sociedad y sus procesos políticos, históricos, económicos y sociales" (Valero et al., 2015).

Al respecto, Skovsmose, Yasukawa y Ravn (2015, citados en Valero et al., 2015) advierten la necesidad de mirar críticamente el conocimiento matemático y de mantener una postura ética de la mano de las matemáticas. De esta visión de las matemáticas, se desprende un compromiso del docente frente a las decisiones que toma, con el reconocimiento de que tales decisiones no son irrelevantes ni inocuas (Scaglia, 2012; English y Watson, 2016).

A la par de dicha postura, la estadística y su educación están también lejos de ser neutrales. Las decisiones políticas y sociales de las naciones se toman con base en la estadística, es decir, en información y datos del entorno, lo que claramente afecta la vida y cultura de las comunidades. Aseveran Vithal y Valero (2012) que, a lo largo de la evolución de nuestras sociedades occidentales, la estadística se ha construido y utilizado como una herramienta de poder en el proceso social, y, además, que la estadística ha sido uno de los pilares de la preminencia de valores como la racionalidad, el objetivismo, el control, la normalización y la abstracción que gobiernan el mundo occidental apoyados por el "auge de la ciencia como conocimiento legítimo que permite tomar las decisiones correctas".

Desrosières (1998) vincula el desarrollo de la estadística y la constitución de Estados modernos como Inglaterra, Alemania y Francia, y señala que la estadística en la práctica se ha usado para describir el poder y la riqueza de los Estados, y teóricamente, como herramienta para abordar la incertidumbre.

Weiland (2019) reconoce el carácter político de la estadística porque los intereses de las personas definitivamente desempeñan un papel en la fabricación e interpretación de argumentos basados en datos y en las decisiones que toman. En esta dirección, Moore (1998) afirma que razonar estadísticamente acerca de los datos, la variación y el azar es un modo de pensar extensible y ampliamente aplicable, que hace prevalecer las cuestiones estadísticas en las discusiones políticas y sociales.

El pensamiento estadístico, argumentan Pfannkuch y Wild (2004), se requiere no solo para extraer información de los datos, sino como una forma de ver la realidad del mundo. Para Weiland $(2017,2019)$, la lectura y escritura del mundo por medio de la estadística posibilita comprender los contextos sociopolíticos e intentar transformarlos. Apunta delMas (2004) que el razonamiento estadístico es necesario para acceder a un mundo objetivo; además, añade este autor, que el trabajo estadístico en torno a situaciones reales tiene un impacto directo en las acciones humanas.

De manera aún más drástica, Batanero (2001) designa la educación estadística como un motor de desarrollo, pues relaciona el progreso de un país con el grado de claridad, completitud y confiabilidad de la información estadística que se produce allí, ya que es con base en esta información que se toman decisiones económicas, sociales y políticas, no solo por parte de los estadísticos, sino de cualquier profesional y ciudadano.

\section{Participación crítica}

Dice Skovsmose (1999a) que la democracia no puede existir sin una capacidad crítica de las personas. Así, la EMC propende por formar una ciudadanía crítica que participe en la vida social y política, a través de desarrollar una competencia democrática (Skovsmose, 1999b; 2012a; 2012b; 2014; 2016). Valero et al. (2015) establecen que esta competencia democrática requiere de cierta competencia en matemáticas, que permita considerar y calificar las 
decisiones públicas que se toman con base en modelos matemáticos; sin embargo, para estos autores la competencia en matemáticas va más allá de los conocimientos matemáticos que muchas organizaciones ${ }^{3}$ promulgan como herramienta necesaria para los ciudadanos en el mercado laboral y económico global, pues señalan que el conocimiento matemático posibilita "cuestionar a las autoridades y, por lo tanto, poder hacer frente a la injusticia" (p. 291).

Entonces, en la EMC la competencia matemática está enraizada en un espíritu de crítica y en posibilidades orientadas hacia la emancipación social (Skovsmose, 1999a) y se vincula "con la posibilidad de crítica social y, con una visión política que vela por el cuestionamiento de las estructuras de poder en la sociedad" (Valero et al., 2015, p. 291). Según Skovsmose y Borba (2004), se constituye en una competencia matemática crítica, que es, de acuerdo con Barwell (2013), un elemento para la formulación de una crítica social o política.

Skovsmose y Valero (2012b) determinan la relación entre educación matemática y democracia por las diferentes dimensiones con las que se puede vincular el conocimiento matemático entendido como poderoso: en un sentido lógico, psicológico, cultural y social. En consecuencia, Valero et al. (2015) advierten que no necesariamente una educación matemática que conduce a la apropiación efectiva del contenido matemático, "es intrínsecamente democrática". Es decir, no toda alusión a la educación matemática como ineludible para la ciudadanía está enmarcada dentro de una visión crítica (Skovsmose, 1999a). En otras palabras, no es posible afirmar que la educación matemática, por sí misma, contribuya a desarrollar ciudadanos críticos con ideales democráticos. De otra parte, tampoco se puede aseverar que el conocimiento matemático, entrañe peligros como, por ejemplo, llevar a posturas dictatoriales o soportar aspectos problemáticos de los procesos sociales (Skovsmose, 2004).

La EMC conecta el desarrollo de la competencia democrática, con un conocer reflexivo que posibilita identificar los rasgos críticos de la sociedad y tomar posiciones justificadas sobre asuntos sociales, políticos y tecnológicos, y reaccionar como ciudadanos críticos en la sociedad de hoy en día; es decir, la competencia democrática hace posible la participación de las personas en los debates y las evaluaciones de las acciones del Gobierno (Skovsmose, 1999a).

Las recomendaciones de la EMC plantean el desarrollo de la capacidad de leer las situaciones que se presentan, con actitud abierta al cambio (Skovsmose, 2012a; 2016), y fundamentan la realización de un proceso educativo crítico, en la disposición de la persona (antecedentes y posibilidades) ligada a su intención (Skovsmose, 1999a). En este sentido, Skovsmose (2004; 2010) recalca las posibilidades divergentes ante la indeterminación de la racionalidad matemática, que pueden ser deliberadas por el aprendiz.

Martínez-Castro (2020) relaciona las ideas de Skovsmose sobre la competencia matemática con la alfabetización estadística crítica propuesta por Weiland (2017), que este investigador describe cómo darle sentido al lenguaje y símbolos estadísticos y criticar información estadística y argumentos con base en datos, para obtener una conciencia de la injusticia y aportar al cambio de las sociedades.

Similarmente, en su idea de alfabetización estadística, Gal (2004) involucra la evaluación crítica de la información después de ser entendida e interpretada. Así, la caracterización de alfabetización estadística de Gal $(2004 ; 2005)$ se refiere a dos componentes interrelacionados: la "habilidad para interpretar y evaluar críticamente información estadística, argumentos

\footnotetext{
3 Por ejemplo, aunque la OECD señala que las matemáticas juegan un papel en la formación de ciudadanos informados y reflexivos, la enseñanza de las matemáticas que promulga se orienta a interpretar información y resolver problemas (Valero et al., 2015).
} 
relacionados con datos o fenómenos estocásticos que puede encontrar en diversos contextos", y la "habilidad para discutir o comunicar sus reacciones a esa información estadística, tales como su comprensión del significado de la información, sus opiniones sobre las implicaciones, - sus dudas acerca de la aceptabilidad de las conclusiones" (2005, p. 49) de manera justificada. Recalca Gal (2004) que para ser "estadísticamente letrado" es necesaria la disposición a formular preguntas que sean el origen de un cuestionamiento crítico y a asumir posturas críticas en términos de creencias y actitudes flexibles.

Otros autores también se refieren a esta actitud crítica requerida al abordar la estadística. Para Batanero et al. (2013), la cultura estadística que se desarrolla al enseñar a través de investigaciones y proyectos permite al ciudadano participar en la sociedad de la información con espíritu crítico e iniciativa personal. Watson (2006) propone como elementos determinantes de la alfabetización estadística, la comprensión de los razonamientos y argumentos estadísticos con una actitud crítica para cuestionar argumentos que estén basados en evidencia estadística. Garfield y Ben-Zvi (2008) enfatizan la necesidad de que todo ciudadano provea argumentos y evalue críticamente conclusiones. Nolan y Speed (1999, citados en Batanero y Díaz, 2011) resaltan la importancia de desarrollar la capacidad discursiva como medio de ampliar habilidades de pensamiento crítico, de manera que el análisis de datos se sitúe dentro de argumentos coherentes y convincentes.

Asimismo, los niveles de alfabetización estadística para un ciudadano, referidos por Watson (2006) y Watson y Callingham (2003, citados en Garfield y Ben-Zvi, 2008), abarcan el desarrollo de una actitud crítica de los resultados promulgados. Las competencias implicadas en estos niveles son sintetizadas por Rumsey (2002) en la habilidad de una persona para desempeñarse en una sociedad basada en datos: ser capaz de consumir la información diaria, pensar críticamente sobre esta y tomar decisiones con base en ella. Esta habilidad, que Rumsey denomina "ciudadanía estadística", debe ser parte integral de la formación de las personas y debe desarrollarse en conjunto con una "competencia estadística", referida al conocimiento acerca de las colecciones de datos, de los conceptos básicos de estadística, y a habilidades de interpretación y comunicación, como antesala de las capacidades de razonar y pensar estadísticamente.

En la misma dirección, Skovsmose señala que "es crucial abordar la estadística desde una perspectiva crítica y simultáneamente reconocer la profunda complejidad de cualquier actividad crítica" (2016, p. 1). Para este investigador, la alfabetización estadística es una característica sustancial de ser un ciudadano crítico, la cual se refiere a desarrollar no solo competencias para realizar cálculos y procedimientos complejos para la vida en sociedad o para la profesión, en un sentido funcionalista sugerido por Apple, sino también a competencias para "aplicar nociones y para reflexionar en la relevancia, la confiabilidad y las limitaciones y sesgos de esas aplicaciones" (1992, p. 65); recuerda, además, que los estudiantes son ciudadanos de una sociedad donde las matemáticas y la estadística se ponen en práctica. Autores como Campos (2016) muestran cómo abordar críticamente asuntos sociales desarrollando, al mismo tiempo, alfabetización estadística.

\section{Papel del docente y de los estudiantes}

De acuerdo con Skovsmose (2012b), la propuesta de la EMC de enmarcar los problemas de la vida real en escenarios de investigación ofrece recursos para la reflexión donde los estudiantes son sujetos activos de su propio proceso de aprendizaje y asumen un papel de investigadores, haciendo de ellos seres que actúan y reflexionan como parte de una sociedad. Con el fin de 
promover la competencia democrática, la EMC concibe el salón de clase como un foro democrático donde las ideas son presentadas y negociadas, y donde se propicia el desarrollo de ciudadanía crítica (Skovsmose y Borba, 2004).

Aunque la EMC privilegia un entendimiento analítico de la educación matemática, igualmente busca pensar y experimentar con las posibilidades de transformar las prácticas educativas existentes (Skovsmose y Borba, 2004). Es claro que para una perspectiva política de la educación matemática es de interés imaginar posibles situaciones nuevas de enseñanza y aprendizaje en el aula que generen un diálogo donde se pueden llevar a cabo relaciones democráticas entre estudiantes y maestros (Skovsmose y Borba, 2004).

La EMC, al promover la desjerarquización del docente dentro del aula y abordar la relación conocimiento-profesor-estudiante desde el diálogo, asocia la educación con un proceso de democratización donde se incita la ruptura de poderes entre maestros y estudiantes (Skovsmose, 2014), en el mismo sentido que el rechazo planteado por Giroux (2005), a las relaciones de subordinación en el salón de clase.

De manera análoga, en la educación estadística la lógica tradicional de la escuela se transforma cuando el foco en los procedimientos estadísticos se transfiere al problema que se trata; la práctica educativa se reorganiza cambiando el papel del profesor y de los estudiantes; la enseñanza y el aprendizaje se vuelven procesos colectivos de investigación en pro de hallar o proponer soluciones a las problemáticas abordadas (Skovsmose, 2016).

Sugieren Batanero y Díaz (2011) y Batanero et al. (2013) que la implementación de proyectos en el aula involucra "a los estudiantes en el ciclo de investigación, modelización y modos propios del razonamiento estadístico" (Batanero et al., 2013, p. 17), contribuye a transponer los conocimientos estadísticos al problema abordado y propicia el desarrollo de competencias sociales y ciudadanas, como la participación y autorresponsabilidad del estudiante en la toma de elecciones y decisiones. Para estos autores, los estudiantes son quienes plantean el problema, deciden sobre los datos y los recolectan, deciden los conceptos y técnicas a utilizar, hacen el análisis y concluyen acerca del problema. Según Cobb y Hodge (2002, citados en Batanero y Díaz, 2011), el trabajo en grupos y la perspectiva sociocultural en la clase de estadística promueve la identificación de los estudiantes como productores de estadísticas con relación a sus propios intereses y problemas.

Así, más que las directrices o la gestión del profesor, lo que suele emerger es un compromiso del estudiante con el desarrollo del proyecto, una conciencia de la importancia de la estadística en la sociedad actual, y de la relevancia de involucrarse en análisis de procesos estadísticos para la mejora de la sociedad. Al dar preferencia a que los proyectos sean planteados por los propios estudiantes, se favorecen igualmente las competencias de autonomía e iniciativa personal, se aviva la capacidad de elegir con criterio propio y se fomenta la ejercitación de la imaginación y las ganas de llevar adelante las acciones requeridas y los planes personales del estudiante, con libertad de elegir las estrategias de resolución, sin depender tanto del profesor (Batanero y Díaz, 2011).

\section{Reflexión final}

Se percibe entonces que algunas de las preocupaciones y pretensiones de la comunidad internacional, y en particular de la EMC, con respecto a la educación son compartidas por el desarrollo reciente de la educación estadística. 
Las coherencias develadas pueden sintetizarse, para empezar, en una afinidad concerniente al reconocimiento de que el desarrollo de la alfabetización, tanto matemática como estadística, es una formación deseada que debe ser accesible para todos; entonces, el trabajo desde estas dos perspectivas se mueve cada vez más a crear posibilidades de acceso y receptividad del conocimiento para todos.

Asimismo, es evidente la coincidencia en ambas disciplinas, por gestionar el proceso de enseñanza y aprendizaje, abordando situaciones reales del entorno social, que sean cercanas y significativas para los estudiantes. Además, es equivalente la postura frente a los saberes que se ponen en juego, pues se considera que las matemáticas y la estadística están lejos de ser neutrales: afectan la vida y cultura de las comunidades dado que son concluyentes en el cuestionamiento y la toma de decisiones políticas y sociales.

También es concordante el carácter crítico involucrado en la educación estadística y la EMC, el cual promueve la integración de las capacidades de ser crítico y de asumir posiciones frente a las situaciones abordadas y los resultados encontrados para crear cambios en la sociedad. De manera similar para las dos perspectivas, la relación conocimiento-profesor-estudiante en el aula se encamina a procesos de democratización donde los papeles de maestros y estudiantes cambian y se emparejan; se establecen diálogos, en los cuales el conocimiento se debate y negocia.

En consecuencia, esta resonancia abre caminos de exploración promisorios para enriquecer el trabajo y la investigación en educación estadística; posibilita, además, pensar en y contribuir a la formulación de una educación estadística que sea crítica, campo de indagación emergente que cuenta con aportes iniciales provenientes del trabajo de autores como Campos (2007, 2016); Arias et al. (2013); Magallanes et al. (2013); Lebrun y Zapata-Cardona (2017); Weiland (2017, 2019).

Este nuevo campo fortalece la posición crítica que surge de forma natural de la estadística como instrumento indispensable para su enseñanza y aprendizaje, teniendo en cuenta el impacto de esta disciplina en el desarrollo sociocultural y político de las sociedades actuales y la necesidad de que estas sean verdaderas comunidades democráticas con miembros activos y participativos en su desarrollo y gestión. Se requiere que la educación estadística forme estudiantes que se sientan libres de pensar e imaginar mundos distintos y que estén familiarizados con el cuestionamiento y la argumentación frente a los resultados que se obtienen y las decisiones que, por consiguiente, se toman.

\section{Referencias}

Aguilar, M. (2014). Educación matemática crítica en México: una argumentación sobre su relevancia. DIDAC, 64, 29-35.

Andrade, L., Fernández, F. y Álvarez, I. (2017). Panorama de la investigación en Educación Estadística desde tesis doctorales 2000-2014. Tecné, Episteme y Didaxis: TED, 41, 87-107.

Apple, M. (1992). Do the Standards go far enough? Power, policy, and practice in Mathematics Education. Journal for Research in Mathematics Education, 23(5), 412431. 
Arias, C., Clavijo, M. y Torres, J. (2013). Fomentando el pensamiento crítico desde el aula estadística. Una propuesta de ambientes de aprendizaje. En R. Flores (ed.), Acta Latinoamericana de Matemática Educativa (pp. 289-298). Comité Latinoamericano de Matemática Educativa.

Azcárate, P. y Cardeñoso, J. (2011). La enseñanza de la estadística a través de escenarios: implicación en el desarrollo profesional. Boletim de Educação Matemática, 24(40), 789-810.

Barwell, R. (2013). The academic and the everyday in mathematicians' talk: the case of the hyperbagel. Journal Language and Education, 27(3), 207-222.

Batanero, C. (2001). Didáctica de la estadística. Grupo de Investigación en Educación Estadística, Universidad de Granada.

Batanero, C. (2002). Los retos de la cultura estadística. Jornadas Interamericanas de Enseñanza de la Estadística.

Batanero, C. (2019). Treinta años de investigación en educación estocástica: reflexiones y desafíos. En J. Contreras, M. Gea, M. López-Martín y E. Molina-Portillo (eds.), Actas del Tercer Congreso Internacional Virtual de Educación Estadística. www.ugr.es/local/fqm126/civeest.html

Batanero, C., Díaz, C., Contreras, J. y Roa, R. (2013). Sentido estadístico y su desarrollo. Números, 83, 7-18.

Batanero, C. y Díaz, C. (2011). Estadística con proyectos. Departamento de Didáctica de la Matemática, Universidad de Granada.

Ben-Zvi, D. y Garfield, J. (2004). Statistical literacy, reasoning and thinking: Goals, definitions and challenges. En D. Ben-Zvi y J. Garfield (eds.), The challenge of developing statistical literacy, reasoning and thinking (pp. 3-16). Kluwer Academic Publishers.

Budgett, S. y Pfannkuch, M. (2007). Assessing students' statistical literacy. Assessment Methods in Statistical Education: An international Perspective, 19, 103.

Campos, C. (2007). A educação estatística: uma investigação acerca dos aspectos relevantes à didática da estatística em cursos de graduação. [Tesis de doctorado, Universidade Estadual Paulista]. Repositorio institucional Unesp http://hdl.handle.net/11449/102161

Campos, C. (2016). Towards Critical Statistics Education. Theory and practice. Lap Lambert Academic Publishing.

Chance, B. (2002). Components of statistical thinking and implications for instruction and assessment. Journal of Statistics Education, 10(3).

Clements, C. (2000). From exercises and tasks to problems and projects: Unique contributions of computers to innovative mathematics education. The Journal of Mathematical Behavior, 19(1), 9-47. 
Cobb, G. (2005). The introductory statistics course: A saber tooth curriculum? Ponencia en United States Conference on Teaching Statistics. Columbus, Ohio.

delMas, R. (2004). A comparison of mathematical and statistical reasoning. En D. Ben-Zvi y J. Garfield (eds.), The challenge of developing statistical literacy, reasoning and thinking (pp. 79-96). Kluwer Academic Publishers.

Denzin, N. y Lincoln, Y. (2015). Métodos de recolección y análisis de datos (Manual de investigación cualitativa IV). Editorial Gedisa.

Desrosières, A. (1998). The Politics of Large Numbers. A History of Statistical Reasoning. Harvard University Press.

Dulzaides, M. y Molina, A. (2004). Análisis documental y de información: dos componentes de un mismo proceso. ACIMED, 12(2).

Eichler, A. y Zapata-Cardona, L. (2016). Empirical Research in Statistics Education. En Empirical Research in Statistics Education. ICME-13 Topical Surveys. Springer, Cham. https://doi.org/10.1007/978-3-319-38968-4_1

English, L. y Watson, J. (2016). Making decisions with data: Are we environmentally friendly? Australian Primary Mathematics Curriculum, 21(2), 3-7.

English, L. (2013). Promoting statistical literacy through data modelling in the early school years. En E. Chernoff y B. Sriraman (eds.), Probabilistic thinking: Presenting plural perspectives (pp. 441-457). Springer.

Fernández, F., Andrade, L. y Álvarez, I. (2016). Panorama de la investigación en Educación Estadística (reporte de investigación). Universidad Pedagógica Nacional.

Fernández, F., Andrade, L. y Álvarez, I. (2018). La investigación en Educación Estadística en el DMA-UPN: una mirada para transformar (reporte de investigación). Universidad Pedagógica Nacional.

Freire, P. (1967). Educación como práctica de libertad. Siglo XXI Editores.

Gal, I. (2004). Statistical literacy. En D. Ben-Zvi y J. Garfield (eds.), The challenge of developing statistical literacy, reasoning, and thinking (pp. 47-78). Kluwer Academic Publishers.

Gal, I. (2005). Towards "probability literacy" for all citizens: Building blocks and instructional dilemmas. En G. Jones (ed.), Exploring probability in school. Challenges for teaching and learning (pp. 39-63). Kluwer Academic Publishers.

Gal, I. (2016). Assessment of adult numeracy skills. Paper commissioned for the Unesco Global Education Monitoring Report 2016: Education for people and planet. http://unesdoc.unesco.org/images/0024/002455/245573E.pdf

Garfield, J., delMas, R. y Zieffler, A. (2010). Assessing statistical thinking. En P. Bidgood, N. Hunt, y F. Jolliffe (eds.), Assessment methods in statistical education: An international perspective (pp. 175-186). John Wiley \& Sons. 
Garfield, J. y Ben-Zvi, D. (2008). Developing students' statistical reasoning. Connecting research and teaching practice. Springer.

Giroux, H. (2005). Against the new authoritarianism. Arbeiter Ring.

Godino, J., Batanero, C. y Font, V. (2007). The onto-semiotic approach to research in mathematics education. ZMD Mathematics Education, 39, 127.

Guidelines for Assessment and Instruction in Statistics Education (GAISE). (2016). College Report ASA Revision Committee. American Statistical Association.

Hassad, R. (2007). Development and validation of a scale for measuring instructors' attitudes toward concept-based or reform-oriented teaching of introductory statistics in the health and behavioral sciences. [Tesis de doctorado, Touro University International]. Repositorio institucional IASE. https://www.stat.auckland.ac.nz/ iase/publications/dissertations/07. Hassad.Disse rtation.pdf

Huff, D. (1954). How to Lie with Statistics. W. W. Norton.

Jablonka, E. (2003). Mathematical Literacy. En A. Bishop, M. Clements, C. Keitel, J. Kilpatrick y F. Leung (eds.), Second International Handbook of Mathematics Education (pp. 75-102). Kluwer Academic Publishers.

Kelly, F. (2019). Connecting disciplinary and pedagogical spaces in statistics: Perspectives from graduate teaching assistants. [Tesis de doctorado, Florida State University]. Repositorio institucional FSU. https://fsu.digital.flvc.org/islandora/object/fsu\%3A709749

Le, L. (2017). Assessing the Development of Students' Statistical Thinking: An Exploratory Study. [Tesis de doctorado, Saint Paul University of Minnesota]. Repositorio institucional UMN. https://hdl.handle.net/11299/185599

Lebrun, V. y Zapata-Cardona, L. (2017). Una perspectiva crítica para la enseñanza de la estadística. XXVII Simposio Internacional de Estadística, 5th International Workshop on Applied Statistics (pp. 1-5). Universidad de Antioquia, Medellín.

Magallanes, A., Colaneri, D. y Rodrígruez, M. (2013). Un escenario de investigación para el desarrollo de una educación estadística crítica en el nivel medio. En J. Contreras, G. Cañadas, M. Gea y P. Arteaga (eds.), Actas de las Jornadas Virtuales en Didáctica de la Estadística, Probabilidad y Combinatoria (pp. 587-593). Universidad de Granada.

Martínez-Castro, C. (2020). Ciudadanía crítica en la formación inicial de profesores de estadística. [Tesis de maestría, Universidad de Antioquia]. Repositorio institucional UdeA.

http://bibliotecadigital.udea.edu.co/bitstream/10495/14473/1/MartinezCastroCind y_2020_CiudadaniaCriticaFormacion.pdf

Ministerio de Educación Nacional (MEN). (1996). Lineamientos curriculares. Matemáticas. Ministerio de Educación Nacional. 
Moore, D. (1992). Teaching statistics as a respectable subject. En F. Gordon y S. Gordon (eds.), Statistics for the twenty-first century (pp. 14-25). Mathematical Associations of America.

Moore, D. (1998). Statistics among the liberal arts. Journal American Statistical Association, 93, 1253-1259.

Moore, D. (1999). Discussion: What shall we teach beginners? International Statistical Review, 67, 250-252.

National Council of Teachers of Mathematics (NCTM). (2000). Principles and standards for school mathematics. NCTM.

Peters, S. (2009). Developing an understanding of variation: AP statistics teachers' perceptions and recollections of critical moments. [Tesis de doctorado, The Pennsylvania State University). IASE. http://www.iaseweb.org/documents/dissertations/16.AneliseGSabbag.Dissertation.pdf

Pfannkuch, M. y Wild, C. (2004). Towards an understanding of statistical thinking. En J. Garfield y D. Ben-Zvi (eds.), The challenge of developing statistical literacy, reasoning and thinking (pp. 17-46). Kluwer Academic Publishers.

Pino del, G. y Estrella, S. (2012). Educación Estadística: relaciones con la matemática. x Congreso Latinoamericano de Sociedades de Estadística. Córdoba, Argentina.

Rumsey, D. (2002). Statistical Literacy as a goal for introductory statistics courses. Journal of Statistics Education, 10(3).

Sabbag, A. (2016). Examining the relationship between statistical literacy and statistical reasoning. [Tesis de doctorado, University of Minnesota]. Repositorio institucional UMN. https://conservancy.umn.edu/handle/11299/182193

Scaglia, S. (2012). Educación matemática crítica. En M. Pochulu y M. Rodríguez (comps.), Educación matemática: aportes a la formación docente desde distintos enfoques teóricos (pp. 201-226). Universidad Nacional de Villa María.

Scheaffer, R. (1997). Discussion-New pedagogy an new content: The case of statistics. International Statistical Review, 65(2), 156-158.

Skovsmose, O. (1999a). Hacia una filosofía de la educación matemática crítica. Una Empresa Docente, Universidad de los Andes.

Skovsmose, O. (1999b). Aporism and Critical Mathematics Education. Universidad de Alborg.

Skovsmose, O. (2004). Critical mathematics education for the future. Conferencia en el $x$ Congreso Internacional en Educación Matemática (ICME).

Skovsmose, O. (2010). Mathematics: A Critical Rationality? Philosophy of Mathematics Education Journal, 25. 
Skovsmose, O. (2012a). Alfabetismo matemático y globalización. En P. Valero y O. Skovsmose (comps.), Educación matemática crítica. Una visión sociopolítica del aprendizaje y la enseñanza de las matemáticas (pp. 65-82). Ediciones Uniandes.

Skovsmose, O. (2012b). Escenarios de investigación. En P. Valero y O. Skovsmose (comps.), Educación matemática crítica. Una visión sociopolítica del aprendizaje y la enseñanza de las matemáticas (pp. 109-130). Ediciones Uniandes.

Skovsmose, O. (2014). Critical Mathematics Education. En S. Lerman (ed.), Encyclopedia of Mathematics Education (pp. 116-120). Springer Netherlands.

Skovsmose, O. (2016). Preface. En C. Campos, Towards Critical Statistics Education. Theory and Practice (pp. 1-4). Lap Lambert Academic Publishing.

Skovsmose, O. y Borba, M. (2004). Research methodology and critical mathematics education. En P. Valero y R. Zevenbergen (eds.), Researching the socio-political dimensions of Mathematics Education: Issues of power in theory and methodology (pp. 207-226). Kluwer Academic Publishers.

Skovsmose, O. y Valero, P. (2012a). Rompimiento de la neutralidad política: el compromiso crítico de la educación matemática con la democracia. En P. Valero y O. Skovsmose (comps.), Educación matemática crítica. Una visión sociopolítica del aprendizaje y la enseñanza de las matemáticas (pp. 1-24). Ediciones Uniandes.

Skovsmose, O. y Valero, P. (2012b). Acceso democrático a ideas matemáticas poderosas. En P. Valero y O. Skovsmose (comps.), Educación matemática crítica. Una visión sociopolítica del aprendizaje y la enseñanza de las matemáticas (pp. 25-64). Ediciones Uniandes.

Spinoza, B. (1999). Ética (trad. Vidal Peña). Alianza.

Valero, P. (1999). Prefacio. En O. Skovsmose, Hacia una filosofía de la educación matemática crítica (pp. viI-XXv). Una Empresa Docente. Universidad de los Andes.

Valero, P. (2012). La educación matemática como una red de prácticas sociales. En P. Valero y O. Skovsmose (comps.), Educación matemática crítica. Una visión sociopolítica del aprendizaje y la enseñanza de las matemáticas (pp. 299-326). Ediciones Uniandes.

Valero, P., Andrade-Molina, M. y Montecino, A. (2015). Lo político en la educación matemática: de la educación matemática crítica a la política cultural de la educación matemática. relime, 18(3).

Vithal, R. y Skovsmose, O. (2012). Mathematics education, democracy and development: A view of the landscape. Revista Pythagoras, 33(2), 1-3.

Vithal, R. y Valero, P. (2012). La investigación en Educación matemática en situaciones de conflicto social y político. En P. Valero y O. Skovsmose (comps.), Educación matemática crítica. Una visión sociopolítica del aprendizaje y la enseñanza de las matemáticas (pp. 217-268). Ediciones Uniandes. 
Watson, J. (2006). Statistical literacy at school: growth and goals. Lawrence Erlbaum Associates.

Weber, V. (2017). Defining the relationship between learning statistical computing and a student's ability to think and reason statistically. [Tesis de doctorado, North Carolina State University). Repositorio institucional NCSU. https://repository.lib.ncsu.edu/bitstream/handle/1840.20/35762/etd.pdf?sequenc $\mathrm{e}=1$ \&isAllowed $=y$

Weiland, T. (2017). Problematizing statistical literacy: An intersection of critical and statistical literacies. Educational Studies in Mathematics, 96, 33-47.

Weiland, T. (2019). Critical Mathematics Education and Statistics Education: possibilities for transforming the school mathematics curriculum. En G. Burrill y D. Ben-Zvi (eds.), Topics and Trends in Current Statistics Education Research. International Perspectives (pp. 391-411). Springer.

Ziegler, A. (2014). Reconceptualizing statistical literacy: Developing an assessment for the modern introductory statistics course. [Tesis de doctorado, University of Minnesota]. Repositorio institucional UMN. https://conservancy.umn.edu/handle/11299/165153 